\title{
Dermatillomanie (Skin-Picking-Störung): Diagnostik, Erklärung und Behandlung
}

L. M. Mehrmann, A. L. Gerlach, A. Hunger

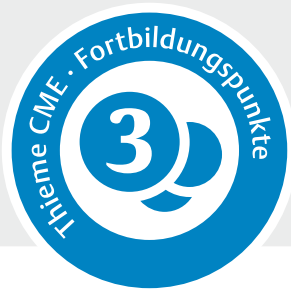

\begin{abstract}
Wenn Menschen ihre Haut durch Knibbeln (Quetschen, Drücken, Kratzen) verletzen und sie damit kaum aufhören können, spricht man von Dermatillomanie (pathologisches Hautzupfen/-quetschen; Skin-Picking-Störung). Diese Angewohnheit wird als befriedigend erlebt, führt zu Leid und ist für Außenstehende schwer nachvollziehbar. Dieser Artikel soll helfen, das Störungsbild zuverlässig zu erkennen, einfühlsam zu verstehen und effektiv zu behandeln.
\end{abstract}

\section{Dermatillomanie - Was ist das?}

Das Bearbeiten der Haut (knibbeln, quetschen, zupfen, drücken, kratzen) ist eine verbreitete menschliche Angewohnheit und nicht per se pathologisch. In begrenztem Maße kann dieses Verhalten für Körperhygiene, Pflege und Entspannung sinnvoll sein. Gemäß Paracelsus macht jedoch die Dosis das Gift. Wenn das Verhalten zu häufig, lange, intensiv, unkontrolliert oder in unangemessenen Situationen auftritt und damit Leiden hervorruft, dann sprechen wir von einer psychischen Störung. Die Bezeichnung „Derma-tillo-manie“ ist griechischen Ursprungs und bedeutet so viel wie „leidenschaftliches Hautzupfen“" $($ Tab. 1$)$.

Menschen mit Dermatillomanie erleben einen unwiderstehlichen Drang, an ihrer Haut zu knibbeln. Trotz wiederholter Versuche schaffen sie es nicht, längerfristig damit aufzuhören. Das übermäßige Knibbeln führt zu erheblichen Hautverletzungen und Schäden, Narben oder tiefen Kratern im Hautbild. Die Betroffenen schämen sich für ihr zerstörtes Hautbild und die erlebte mangelnde Willensstärke. Sie leiden unter dem unwiderstehlichen Drang zu knibbeln, reagieren mit Schuldgefühlen und Selbstabwertung. Diese langfristig negativen Konsequenzen des Knibbelns können den privaten und beruflichen Alltag der Betroffenen schwer beeinträchtigen. Sowohl das Knibbeln als auch die gedankliche Beschäftigung mit diesem Thema kann im Leben der Betroffenen beeinträchtigend viel Zeit beanspruchen.

\begin{abstract}
Merke Menschen mit Dermatillomanie sind hin- und hergerissen zwischen dem als befriedigend erlebten Knibbeln an der Haut und der Vermeidung langfristig schädigender Konsequenzen. Sie leiden unter diesem intrapsychischen Annäherungs-Vermeidungs-Konflikt.
\end{abstract}

\section{Definition und Klassifikation}

Dermatillomanie ist bislang nur im Diagnostischen und Statistischen Manual Psychischer Störungen (DSM-5) der American Psychiatric Association explizit definiert worden [1]. In der deutschen Übersetzung ist die englische Bezeichnung Excoriation (Skin Picking) Disorder mit dem sperrigen Begriff „Dermatillomanie (pathologisches Hautzupfen/-quetschen)“ übersetzt worden.

- Tab. 1 Gebräuchliche Bezeichnungen für Dermatillomanie.

\begin{tabular}{|l|l|}
\hline Deutsch & Englisch \\
\hline Dermatillomanie & Dermatillomania \\
\hline pathologisches Hautzupfen/-quetschen & Pathologic Skin Picking \\
\hline chronisches Hautzupfen/-quetschen & Chronic Skin Picking \\
\hline Skin-Picking-Störung & Skin Picking Disorder \\
\hline Exkoriationsstörung & Excoriation Disorder \\
\hline neurotische Exkoriation & Psychogenic/Neurotic Excoriation \\
\hline franz:: Acne excoriée (des jeunes filles) & Grooming Disorder \\
\hline
\end{tabular}




\begin{abstract}
Merke
Im Deutschen benutzen die Betroffenen häufig den englischen Begriff Skin Picking, wenn sie über ihr pathologisches Verhalten sprechen.
\end{abstract}

Im DSM-5 wird Dermatillomanie als eine der Zwangsstörung verwandte Störung eingeordnet. Es werden 5 diagnostische Kriterien für Dermatillomanie festgelegt, die eine zuverlässige Diagnose ermöglichen sollen:

- 2 Kriterien definieren die spezifische Symptomatik: (A) das wiederkehrende, schädliche Knibbeln an der Haut und (B) wiederholte Kontrollversuche.

- Das 3. Kriterium (C) benennt den daraus resultierenden Leidensdruck bzw. die Beeinträchtigung in klinisch bedeutsamer Weise.

- 2 weitere Kriterien enthalten Informationen zu notwendigen differenzialdiagnostischen Abwägungen: (D) ursächlicher Substanzeinfluss/medizinischer Krankheitsfaktor sowie (E) eine andere ursächliche psychische Störung.

In der aktuell gültigen Internationalen Klassifikation der Krankheiten und verwandter Gesundheitsprobleme (ICD-10) der Weltgesundheitsorganisation (WHO) wird Dermatillomanie nicht explizit genannt [2]. Implizit ist das Störungsbild aber an 2 verschiedenen Stellen in der ICD-10 enthalten.

- Im Kapitel F63 kann Dermatillomanie unter „Abnorme Gewohnheiten und Störungen der Impulskontrolle“ diagnostiziert werden. Störungen der Impulskontrolle sind gekennzeichnet durch „das Versagen, dem Impuls, Trieb oder der Versuchung zu widerstehen, eine Handlung auszuführen, die für die Person selbst oder für andere schädlich ist. " [3], S. 691. Unter F63 sind pathologisches Spielen, pathologische Brandstiftung, pathologisches Stehlen sowie Trichotillomanie (Unfähigkeit, Impulsen des Haareausreißens zu widerstehen) aufgeführt. Die

\section{HINTERGRUNDWISSEN}

\section{Definition Dermatillomanie (pathologisches Hautzupfen/-} quetschen) nach DSM-5 [1]

A. Wiederkehrendes Zupfen/Quetschen der Haut, was Hautverletzungen zur Folge hat.

B. Wiederholte Versuche, das Hautzupfen/-quetschen einzuschränken oder zu unterlassen.

C. Das Hautzupfen/-quetschen verursacht in klinisch bedeutsamer Weise Leiden oder Beeinträchtigungen in sozialen, beruflichen oder anderen wichtigen Funktionsbereichen.

D. Das Hautzupfen/-quetschen ist nicht Folge der physiologischen Wirkung einer Substanz (z. B. Kokain) oder eines medizinischen Krankheitsfaktors (z. B. Scabies).

E. Das Hautzupfen/-quetschen kann nicht besser durch eine andere psychische Störung (unter anderem Verletzungsabsicht) erklärt werden.
Kategorie F63.8 „Sonstige abnorme Gewohnheiten und Störungen der Impulskontrolle“ ist vorgesehen für alle noch nicht explizit benannten „Arten von sich dauernd wiederholenden unangepassten Verhaltens, welche nicht Folge eines anderen psychischen Syndroms sind. Die betroffene Person scheint immer wieder den Impulsen, sich auf eine bestimmte Art zu verhalten, nicht widerstehen zu können. Der Handlung geht eine Anspannung voraus, der während des Handlungsablaufs ein Gefühl der Erleichterung folgt.“ [2], S. 162.

- Darüber hinaus ist Dermatillomanie auch als L98.1 „Dermatitis factitia“ (inkl. neurotische Exkoriation) diagnostizierbar. Hier ist die Störung als „Sonstige Krankheit der Haut und der Unterhaut, anderenorts nicht klassifiziert" eingeordnet.

Die genannten Diagnosemöglichkeiten F63.8 und L98. 1 schließen sich gegenseitig aus, dürfen also nicht gemeinsam vergeben werden.

\section{Merke}

Für Dermatillomanie kann der Diagnoseschlüssel F63.8 „Sonstige abnorme Gewohnheiten und Störungen der Impulskontrolle“ oder L98.1 „Dermatitis factitia" verwendet werden.

Die von der WHO vorbereitete Revision ICD-11 wird Dermatillomanie vermutlich explizit enthalten. Im Internet kann man den aktuellen Betaentwurf einsehen unter: apps.who.int/classifications/icd11/browse/l-m/ en\#

In der zuletzt verfügbaren englischen Version (Stand 12/2016) wird „Excoriation Disorder“ (Skin Picking Disorder, Dermatillomania) in der Kategorie „Body-focused repetitive behaviour disorders“ aufgeführt, die unter "Obsessive-compulsive or related disorders" zu finden sind. Die Diagnosekriterien für Dermatillomanie in der ICD-11 werden vermutlich denen des DSM-5 weitestgehend entsprechen.

\section{Wie zeigt sich Dermatillomanie in der Praxis?}

Allen Betroffenen ist der übermäßige Drang zum Knibbeln an der Haut und der daraus resultierende Leidensdruck bzw. die Beeinträchtigung gemeinsam. Dabei können die betroffenen Hautstellen, die Art des Knibbelns, die konkreten Auslöser und die daraus resultierenden Konsequenzen vielfältig sein und im Verlauf der Erkrankung fluktuieren.

\section{Art und Weise des Knibbelns}

Knibbeln dient als Sammelbegriff für verschiedenste Verhaltensweisen, die auftreten können. Die Haut kann durch Quetschen, Kratzen, Zupfen, Drücken oder auch jede andere denkbare Form des Knibbelns geschädigt werden. Die meisten Betroffenen quetschen 
und drücken mit Fingern bzw. Fingernägeln an Hautunreinheiten. Zusätzlich sind Kratzen bzw. Zupfen an Wundschorf häufig. Neben den Fingern können Pinzetten, Nadeln, Drahtschlaufen, Scheren oder andere spitze bzw. scharfe Gegenstände zum Einsatz kommen [4]. Die Haut am Nagelbett und den Fingerkuppen wird zumeist mit den Zähnen abgebissen und anschließend ausgespuckt oder geschluckt.

\section{Betroffene Hautregionen}

Besonders häufig wird im Gesicht geknibbelt. Grundsätzlich können aber alle Hautregionen betroffen sein, vor allem Kopfhaut, Arme, Beine, Dekolleté, Finger und Hände, Rücken. Der Schambereich, Füße und Ohren sind seltener betroffen [5].

\section{Merke}

Eine Person kann an mehr als einer Körperstelle gewohnheitsmäßig knibbeln und die bevorzugte Hautregion kann im Verlauf der Erkrankung wechseln.

\section{Auslöser für das Knibbeln (Trigger)}

Hautunregelmäßigkeiten wie Hautirritationen, Läsionen, Pickel, Insektenstiche, hervorstehende Nagelhaut, Hornhaut oder Wundschorf lösen häufig den Drang zum Knibbeln aus. Hier steht in der Regel die Absicht im Vordergrund, die Haut zu glätten. Aber auch an unauffälliger Haut kann gewohnheitsmäßig geknibbelt werden [4-6]. Zusätzlich zum bewussten Knibbeln mit der gezielten Suche nach „geeigneten“ Hautunregelmäßigkeiten kann das Knibbeln auch unbewusst, d.h. automatisiert und vom Betroffenen unbemerkt, auftreten [4]. Viele Betroffene bemühen sich, nicht in Anwesenheit anderer Personen oder zumindest weitestgehend unbeobachtet zu knibbeln.

\section{Merke}

Am häufigsten ist die Gesichtshaut betroffen.

Für manche Betroffene gehört das Knibbeln zur täglichen Routine, die im Rahmen der Körperpflege morgens und abends vor dem Badezimmerspiegel stattfindet. Abends tritt das Knibbeln oft besonders stark auf, wenn die Zeit nicht durch andere Aktivitäten begrenzt ist. Zum einen werden Hautunregelmäßigkeiten in diesen Situationen häufiger visuell oder taktil wahrgenommen. Zum anderen können die genannten Tätigkeiten im Allgemeinen gleichzeitig mit Knibbeln ausgeführt werden.

Merke

Typische Auslöser für Knibbeln sind Hautunregelmäßigkeiten, kontemplative Tätigkeiten und unangenehme Gefühle.

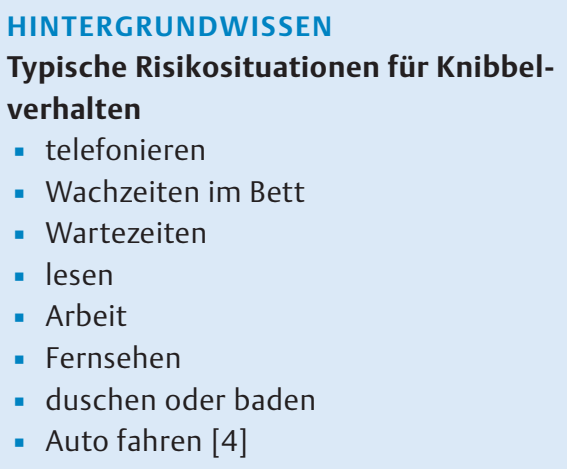

\section{Unmittelbare Auswirkungen des Knibbelns}

Knibbeln an der Haut kann einerseits entspannen, andererseits auch anregen und damit sowohl Unter- als auch Überstimulation ausgleichen. Entsprechend tritt es nicht nur unter emotionaler Anspannung und Stress auf (z.B. Zeitdruck, Entscheidungsschwierigkeiten), sondern auch z. B. bei Langeweile [7]. Betroffene können beim Knibbeln einen dissoziativen Zustand erleben und damit ihre Außenwelt und belastende Gedanken vorübergehend ausblenden. Ein vermindertes Zeitgefühl kann dazu beitragen, dass Knibbeln länger andauert als ursprünglich beabsichtigt. Eine Episode kann dementsprechend wenige Minuten bis zu mehreren Stunden in Anspruch nehmen, mit Schwankungen in der Anzahl der Episoden pro Tag [8]. Betroffene wenden darüber hinaus viel Zeit (und Energie) auf, um gegen den starken Drang zu knibbeln anzukämpfen oder darüber nachzugrübeln. Schwer Betroffene können so nahezu den gesamten Tag mit Knibbeln, dem Versuch zu widerstehen und Gedanken daran verbringen.

Merke

Dermatillomanie kann sowohl die körperliche und psychische Gesundheit als auch die soziale und schulische/berufliche Leistungsfähigkeit beeinträchtigen.

\section{Negative Auswirkungen von Dermatillomanie}

Neben den bereits genannten schädlichen Auswirkungen des übermäßigen Knibbelns auf die Haut und das Selbstbild können als Konsequenz auch finanzielle Einbußen, signifikante Leistungsbeeinträchtigungen sowie zusätzliche psychische Störungen auftreten [8,9]. Die selbst verursachten Hautverletzungen werden aus Scham in der Regel mit langärmeliger und hochgeschlossener Kleidung bzw. Make-up o. Ä. kaschiert. Die aufwendige Vertuschung des angerichteten Schadens kostet Zeit und Geld. Zudem vermeiden Betroffene häufig Aktivitäten, bei denen die Hautschäden für andere sichtbar werden könnten, unter anderem Treffen an hell erleuchteten Orten, formelle Veranstaltungen oder bestimmte Sportarten wie Schwimmen. Auch intime Beziehungen und sexuelle Aktivitäten können we- 


\section{FALLBEISPIEL}

\section{Symptomatik und Verlauf}

Frau Z. stellt sich mit schweren Hautläsionen vor. Erst auf Nachfrage schildert sie zögerlich, ihre Haut aufzuknibbeln und damit kaum aufhören zu können. Bereits in der Kindheit habe sie an Wundschorf geknibbelt. Ihre Mutter habe dann mit ihr geschimpft. Während der Pubertät habe sie regelmäßig ihre Pickel ausgedrückt und damit deutlich mehr Zeit verbracht als andere. Sie habe ihre Haut kaum in Ruhe lassen können. Anfang 20 habe ein Hautarzt sie auf die schädliche Wirkung ihres Verhaltens hingewiesen. Eine Aknecreme habe die entzündeten Pickel reduziert, sie habe aber weiterhin geknibbelt. Aufgrund negativer Reaktionen anderer verheimliche sie ihr Knibbeln. Mit Mitbewohnern kam es zu Konflikten, weil sie zu lange das Bad blockierte. Auch beim Lernen fürs Studium knibbele sie unbewusst ihre Haut im Gesicht auf. Heute verbringe sie proTag ca. 1 Stunde mit Knibbeln. Nicht immer seien offensichtliche Hautunreinheiten vorhanden, auch Wundschorf und andere Unregelmäßigkeiten seien Auslöser.

gen des Knibbelns beeinträchtigt sein. Wichtige Arztbesuche werden möglicherweise aus Scham aufgeschoben.

Betroffene berichten zudem von Schwierigkeiten, konzentriert zu arbeiten, sowie über Probleme bei der Einhaltung von Verpflichtungen während der schulischen/ beruflichen Ausbildung, beispielsweise einen Vortrag zu halten. Fehltage aufgrund des Knibbelns sind selten, aber die Erkrankung kann die Betroffenen davon abhalten, ihre beruflichen Ziele zu verfolgen - wie etwa eine Beförderung oder eine Gehaltserhöhung anzustreben. Ein Großteil der Betroffenen berichtet außerdem von Depressionen und Ängsten sowie der Einnahme legaler oder illegaler Substanzen, um negative Gefühle infolge des Knibbelns zu reduzieren [8].

\section{Epidemiologische Erkenntnisse zu Dermatillomanie}

Da Dermatillomanie bis zum Jahr 2013 in keinem anerkannten Klassifikationssystem explizit definiert wurde, unterscheiden sich die bisherigen Studien zum Teil erheblich in den jeweils zugrunde liegenden Diagnosekriterien. Epidemiologische Kennwerte sind entsprechend vorsichtig zu interpretieren und nur als grobe Schätzungen zu verstehen.

Die Angaben zur geschätzten Lebenszeitprävalenz für Dermatillomanie bewegen sich bei 1,4-5,2\% [5]. In den bisherigen klinischen Studien liegt der Anteil weiblicher Teilnehmerinnen überwiegend bei mehr als $70 \%$ [10]. Der Erkrankungsbeginn liegt häufig zwischen dem 12. und 16. Lebensjahr $[8,11]$. Hier fällt vor allem der zeitlich gemeinsame Beginn von pubertärer Akne auf.

\begin{abstract}
Merke
Schätzungsweise 1 - 5\% der Bevölkerung erkrankt im Lauf ihres Lebens an Dermatillomanie, vor allem Frauen. Die Erkrankung beginnt häufig in der Pubertät und nimmt unbehandelt oft einen chronischen Verlauf mit fluktuierender Symptomatik [6].
\end{abstract}

\section{Komorbide Störungen}

Dermatillomanie kann gleichzeitig mit anderen psychischen Störungen auftreten. Dabei kann sich die Dermatillomanie infolge einer anderen psychischen Störung entwickeln oder die Entstehung einer anderen psychischen Störung begünstigen. Komorbide Störungen können aber auch unabhängig voneinander bestehen, weil sie auf einen gemeinsamen Risiko- oder Ursachenfaktor zurückgehen. In der Literatur werden Zusammenhänge zwischen Skin-Picking-Symptomen und affektiven sowie Angstsymptomen berichtet [5, 12]. Bis dato fehlen zuverlässige Erhebungen zu komorbiden Erkrankungen bei Dermatillomanie.

\section{Diagnostik}

Da Dermatillomanie sehr schambesetzt ist und starke Schuld- und Minderwertigkeitsgefühle auslösen kann, wird das Knibbeln oft verheimlicht und selten offen angesprochen. Es bedarf dementsprechend einer einfühlsamen, direkten Ansprache seitens des Diagnostikers in einer vertrauensvollen und wertschätzenden Gesprächsatmosphäre, um die Symptomatik zuverlässig diagnostizieren zu können. Das Risiko falsch negativer Urteile ist groß, auch weil komorbide Diagnosen mit einem höheren Bekanntheitsgrad schnell in den Vordergrund rücken.

\section{PRAXIS}

Einfache Screeningfragen können das Risiko, Dermatillomanie zu übersehen, deutlich reduzieren:

- „Haben Sie die Angewohnheit, an Ihrer Haut zu knibbeln?"

- „Fällt es Ihnen schwer, Ihre Haut in Ruhe zu lassen bzw. mit dem Knibbeln aufzuhören?“

\section{Diagnostische Interviewverfahren}

Derzeit gibt es noch kein strukturiertes diagnostisches Interview zur Erfassung von Dermatillomanie entsprechend der DSM-5-Kriterien. Die diagnostische Abklärung erfolgt demnach am besten durch ein freies klinisches Interview. Hier bieten sich die oben genannten Einstiegsfragen sowie Fragen zur gezielten Abklärung der weiteren DSM-5-Kriterien an. Darüber hinaus stehen einzelne deutschsprachige Fragebogeninstrumente zur Verfügung, um das Ausmaß der Symptomatik zu quantifizieren. 


\section{Selbstbeurteilungsfragebogen}

Im Deutschen stehen derzeit 2 an einer klinischen Onlinestichprobe validierte Fragebögen für Dermatillomanie zur Verfügung. Die modifizierte Skin-PickingSkala (mSPS-D) [13] erfasst auf 3 Unterskalen die „Häufigkeit“, die „Intensität“ und das Ausmaß von „Leiden und Beeinträchtigung“ des Knibbelns ( $\alpha=.76-.80)$. Die psychosoziale Beeinträchtigung infolge des Knibbelns wird mit der Kurzversion der deutschen Skin Picking Impact Scale (SPIS-D [13]) erfasst. Diese Skala besteht aus 4 Items $(\alpha=.81)$. Eine deutsche Übersetzung der ursprünglichen Skin Picking Scale wurde zudem von Gallinat und Kollegen mit einer nicht selektierten Onlinestichprobe untersucht [14]. SPIS-D und mSPS-D stehen auf Anfrage bei der Erstautorin zur Verfügung.

\section{PRAXIS}

\section{Die deutsche Skin Picking Impact Scale}

(SPIS-D [13])

Bitte kreuzen Sie an, wie sehr Sie die jeweilige Aussage in der vergangenen Woche belastet hat.

[Antwortskala: 0 = gar nicht bis 4 = schwer]

- Ich denke, mein soziales Leben wäre besser, wenn ich nicht knibbeln würde.

- Ich schäme mich wegen meines Skin Pickings.

- Es gibt manche Dinge, die ich wegen meines Skin Pickings nicht machen kann.

- Ich fühle mich unattraktiv wegen meines Skin Pickings.

\section{Differenzialdiagnostische Überlegungen}

Das Risiko einer falsch positiven Dermatillomaniediagnose ist gegeben, wenn pathologisches Knibbeln im Rahmen einer anderen Erkrankung auftritt [15]. Ein eigenständiges Störungsbild ist das pathologische Knibbeln nur dann, wenn es unabhängig vom Vorliegen einer komorbiden Erkrankung erscheint bzw. nicht besser durch eine andere Erkrankung erklärbar ist.

\section{Ursächliche körperliche Erkrankungen} und Substanzkonsum

Bei der diagnostischen Abklärung ist auszuschließen, dass pathologisches Knibbeln lediglich im zeitlichen Zusammenhang mit dem Konsum einer oder mehrerer Substanzen auftritt (z. B. Kokainkonsum). Zudem sollte das Knibbeln nicht ausschließlich infolge von körperlichen Erkrankungen auftreten, wie z. B.

- Hautinfektionen (Pilze, Viren, Bakterien, Milben),

- einer juckreizauslösenden Krankheit (z. B. Neurodermitis) oder

- Autoimmunerkrankung (z. B. Psoriasis).
In den oben genannten Fällen sollte die Grunderkrankung bzw. das Zustandsbild nach Substanzkonsum diagnostiziert und behandelt werden.

\section{Symptom anderer psychischer Erkrankungen}

Wenn pathologisches Knibbeln als Symptom einer anderen psychischen Erkrankung auftritt, ist Dermatillomanie nicht zu diagnostizieren. Beispielsweise können spezifische Wahnvorstellungen (z.B. Dermatozoenwahn) oder (taktile) Halluzinationen im Rahmen einer psychotischen Störung übermäßiges Knibbeln auslösen.

Im Kontext der körperdysmorphen Störung wird übermäßiges Knibbeln primär eingesetzt, um einen vermeintlichen Defekt oder Mangel in der äußeren Erscheinung zu beheben, wohingegen bei Dermatillomanie der Akt des Knibbelns selbst im Vordergrund steht.

Übermäßiges Knibbeln kann auch mit einer gezielten Selbstverletzungsabsicht auftreten, z. B. im Rahmen einer Borderline-Persönlichkeitsstörung. Im Rahmen einer Zwangsstörung kann Knibbeln zur Neutralisierung von Zwangsgedanken eingesetzt werden (z.B. Knibbeln als zwanghafte Reinigungsmaßnahme, etwa um Ansteckung zu verhindern). Im Rahmen einer stereotypen Bewegungsstörung wäre übermäßiges Knibbeln denkbar als repetitive Verhaltensweise zusätzlich zu anderen rhythmischen Bewegungen, wie z. B. wiegende Körperbewegungen und Kopfanschlagen.

\section{Merke \\ Im Unterschied zu möglichen Differenzialdiagnosen steht bei Dermatillomanie das pathologische Knibbeln für die Betroffenen im Vordergrund.}

Bei den genannten Differenzialdiagnosen dient das Knibbeln jeweils als Mittel zu einem bestimmten Zweck. So wird ein Psychotiker verschiedene Verhaltensweisen zeigen, um seinem Wahn bzw. Halluzinationen zu begegnen. Die eingesetzten Verhaltensweisen sind dabei flexibel austauschbar - wichtig ist allein, dass das übergeordnete Ziel erreicht werden kann (z. B. die vermeintlichen Fremdkörper unter der Haut loszuwerden). Bei der körperdysmorphen Störung werden vielgestaltige Maßnahmen eingesetzt, um die störungsspezifischen dysfunktionalen Überzeugungen und Ängste (z. B. aufgrund von Perfektionismus) zu verringern. Gleiches gilt für Zwangsstörungen (wie oben bereits dargestellt). Bei der stereotypen Bewegungsstörung steht der Lustgewinn im Vordergrund, der aus der stereotypen Ausführung des Knibbelns resultiert. Bei primärer Selbstverletzung steht die Verletzung selbst bzw. das Gefühl von Schmerzen oder etwa der Anblick des Blutes im Vordergrund. Dieses Ziel kann wie bei der stereotypen Bewegungsstörung durch völlig unterschiedliche Verhaltensweisen erreicht werden, unter anderem durch Aufkratzen der Haut. 


\section{PRAXIS}

\section{Differenzialdiagnostische Klärung}

Tritt pathologisches Knibbeln im Rahmen von Dermatillomanie auf oder als Symptom einer anderen Erkrankung, z. B. als Bewältigungsverhalten bei Akne? Folgende Fragen können zur differenzialdiagnostischen Klärung beitragen:

„Stellen Sie sich vor, wir würden einen Weg finden, Ihr Hautbild zu Ihrer Zufriedenheit zu verändern, z. B. mit einer professionellen Gesichtsreinigung von einem Experten. Denken Sie, dass wir Ihr Problem so insgesamt lösen könnten? Oder würde es Ihnen trotzdem schwerfallen, nicht mehr zu knibbeln, bzw. Ihnen das Knibbeln fehlen?“

Für Betroffene mit Akne oder körperdysmorpher Störung wäre das Knibbeln bei einem reinen Hautbild unnötig geworden. Für Dermatillomaniebetroffene wäre diese Lösung - im wahrsten Sinne des Wortes - „unbefriedigend“.

Bei Dermatillomanie erfolgt das Knibbeln hingegen um seiner selbst willen: Es entsteht ein ganz spezifischer, unwiderstehlicher Drang, der nicht so leicht durch andere Verhaltensweisen ersetzt werden kann.

\section{Ätiologie}

Warum entwickeln Menschen Dermatillomanie? Grundsätzlich gilt - wie bei anderen Erkrankungen auch -, dass eine ganze Reihe verschiedener Faktoren (biologisch, psychologisch, sozial) im Sinne eines Risikomodells der Dermatillomanie zusammenwirken, damit diese Störung entsteht und aufrechterhalten wird. Ob explizit ein relevanter Stressor zu Beginn der Störung zu identifizieren ist oder ob eher eine schleichende Entwicklung das individuelle Störungsbild kennzeichnet, ist in der individuellen Diagnostik herauszuarbeiten.

\section{Biologische Einflussfaktoren}

Biologische Vulnerabilitätsfaktoren für Dermatillomanie sind noch wenig erforscht. Unbestritten fungieren biologische Faktoren als Auslöser für Knibbeln. So gilt die Pubertät mit ihren typischen neurobiologischen und äußeren körperlichen Veränderungen als Risikophase für die Ersterkrankung. Darüber hinaus kann das Ausmaß des pathologischen Knibbelns mit dem Menstruationszyklus fluktuieren [4].

\section{Genetische Einflussfaktoren}

In bisherigen Studien gaben 3-45\% der befragten Betroffenen an, von mindestens einem Verwandten 1. Grades mit Skin Picking zu wissen [7]. Dieser Hinweis auf eine mögliche familiäre Häufung von Dermatillomanie lässt allerdings keine eindeutigen Rückschlüsse auf eine genetische Veranlagung zu. Solche Häufungen können ebenso auf Lernen (am Modell) und/oder familiär übereinstimmende Umgebungsbedingungen zu- rückgehen. Systematische genetische Familien-/Analogstudien fehlen noch.

\section{Neurobiologische und neuropsychologische Befunde} Eine Reihe von Studien verglich Dermatillomaniebetroffene mit Kontrollpersonen und/oder Trichotillomaniebetroffenen. Strukturelle MRT-Untersuchungen sowie Messungen der Diffusionsbewegungen der weißen Materie lassen Rückschlüsse auf die kortikale Verdichtung in einzelnen Hirnarealen zu. Personen mit Dermatillomanie weisen im Vergleich zu Kontrollpersonen und Personen mit Trichotillomanie eine höhere kortikale Verdichtung des ventralen Striatums auf. Dies weist auf eine Beteiligung des Belohnungssystems am Störungsgeschehen hin [16]. Gleichzeitig zeigen sich im Vergleich zu Kontrollprobanden Auffälligkeiten in der Konnektivität von Bereichen, die relevant für die Bewegungsbildung und -unterdrückung sind (vor allem Verbindungen von und zum anterioren cingulären Kortex) [17]. Zu diesen Ergebnissen passen auch Befunde aus neuropsychologischen Untersuchungen. Hier reagierten Personen mit Dermatillomanie in einer StoppSignal-Reaktionsaufgabe verzögert bei der Unterdrückung von Antwortimpulsen [18].

Die bisher gefundenen Auffälligkeiten bei Dermatillomanie scheinen mit der Phänomenologie der Erkrankung gut vereinbar. Die Betroffenen leiden nach eigenen Angaben insbesondere darunter, dass sie dem reizvollen Drang zum Knibbeln nur schwer widerstehen können. Ist dieses befriedigende Verhalten erst einmal initiiert, ist es den Selbstberichten zufolge schwer, dieses zeitnah abzubrechen. Diese Hürde scheint nicht primär auf ein Motivationsdefizit zurückzugehen, da den Betroffenen die negativen Konsequenzen nach eigenen Angaben durchaus bewusst sind - auch im Moment des Knibbelns selbst.

\section{Ethologische Perspektive}

Verhaltensbiologisch besteht die Vermutung, dass exzessives Körperpflegeverhalten als frühe Form der Stressbewältigung zu verstehen ist $[19,20]$. Als Analogie sind dabei Übersprungshandlungen bei Tieren anzuführen, die Pflegeverhaltensweisen ähneln können und eine Abreaktion in Konfliktsituationen darstellen. Dieser Perspektive zufolge besteht eine generelle biologische Bereitschaft, in Belastungssituationen exzessives Körperpflegeverhalten zu zeigen, um eine spontane Stressreduktion zu erreichen. Dies könnte erklären, warum Beginn und Fluktuationen im Ausmaß des pathologischen Knibbelns häufig mit psychischen und körperlichen Belastungen einhergehen.

\section{Merke}

Das Knibbeln an der Haut kann als eine simple, kurzzeitig effektive Maßnahme zur Emotionsregulierung dienen. 


\section{Soziokulturelle Einflussfaktoren}

Soziale Faktoren haben vor allem aufgrund des menschlichen Bedürfnisses nach Zuneigung und Zugehörigkeit zu einer sozialen Gruppe großen Einfluss: Wir wollen für andere attraktiv sein. Wie attraktiv ein Mensch bewertet wird, hängt stark von äußeren Faktoren ab - und dabei spielt unser Hautbild eine wichtige Rolle [21]. Hautunregelmäßigkeiten sind vor allem im Gesicht besonders offensichtlich und gelten im Allgemeinen als wenig attraktiv. Gleichzeitig sind dort Hautveränderungen aufgrund der sensiblen Beschaffenheit der Gesichtshaut am häufigsten. Zeigen sich während der Pubertät plötzlich vermehrt Hautunreinheiten, so scheint die Akzeptanz durch die soziale Gruppe bedroht, unter anderem weil Menschen sich von (ansteckenden) Krankheiten intuitiv fernhalten. Aus Furcht vor Ablehnung und negativen Fremdurteilen können betroffene Personen dementsprechend vermehrt bereit sein, (übermäßig) an der Haut zu knibbeln.

Gesellschaftliche Einflüsse zeigen sich vor allem in geltenden kulturellen Normen und Werten. Betrachtet man die Darstellung von Haut in den Massenmedien, so wird diese meist mit Fotobearbeitungsprogrammen als vollständig makel- und faltenlos dargestellt. Reine, makellose Haut gilt dementsprechend als (vermeintlich erreichbares) Ideal. Überhöhte Ansprüche an das eigene Hautbild sind die Folge und vergrößern die Unzufriedenheit mit der eigenen wahrgenommenen Attraktivität. Beides kann die Angewohnheit, an der eigenen Haut zu knibbeln, ungünstig verstärken.

In einer australischen qualitativen Studie beurteilten Männer und Frauen mit Akne, Ekzemen und Psoriasis eine makellose Haut als gesellschaftliches Schönheitsideal, was vor allem durch die in den Medien präsentierte, perfekte Darstellung der Haut vermittelt würde. Das Nichterreichen dieses Ideals war nach Angaben der Studienteilnehmerinnen ein Grund für eine erhöhte Rate an psychischen Erkrankungen [22].

\section{Psychologische Faktoren}

Biologische und umweltbedingte Faktoren interagieren mit psychologischen Einflussfaktoren und bestimmen die individuelle Vulnerabilität sowie das Stressniveau. Entsprechend zieht man auch psychologische Lerntheorien und kognitive Erklärungsansätze heran, um die Entstehung und Aufrechterhaltung von Dermatillomanie zu erklären.

\section{Entstehung einer Angewohnheit}

Im Sinne des operanten Konditionierens wiederholen wir Verhaltensweisen, die einen positiven Effekt haben (z.B. angenehm sind). Wie bereits dargestellt, kann Körperpflegeverhalten wie Knibbeln an der Haut eine kurzfristige emotionsregulierende Wirkung haben.
Das Bearbeiten der Haut wird von vielen als reinigend empfunden und ist mit kleinen „Erfolgen“ verbunden, wenn die Haut beim Ausquetschen sichtbar von Talg oder Eiter befreit werden kann. Zusätzlich kann das Bearbeiten der Haut kurzfristig helfen, unangenehme Gefühle zu reduzieren (negative Verstärkung). Hier können dissoziative Zustände oder auch die intensive Konzentration auf die Haut und das Knibbeln dazu beitragen, Übererregung abzubauen und negative Gedanken/Gefühle in den Hintergrund rücken zu lassen. Andererseits kann Knibbeln bei Langeweile auch als anregende Stimulation empfunden werden.

\footnotetext{
Merke

Während des Knibbelns treten vielfältige angenehme Konsequenzen unmittelbar und zuverlässig ein oder reduzieren sich negative Zustände, wodurch das Verhalten verstärkt und somit in Zukunft wahrscheinlicher wiederholt wird.
}

Es ist zu vermuten, dass diese knibbelbezogene positive und negative Verstärkung besonders viel bedeutet für Menschen mit einer hohen Vulnerabilität für Dermatillomanie. Zudem befinden sie sich möglicherweise in Lebensumständen (z. B. chronischer Stress), in denen die oben genannten Konsequenzen einen hohen situativen Verstärkerwert aufweisen. Diese individuellen und situativen Unterschiede können erklären, warum nicht alle Menschen erkranken und warum die Erkrankung in bestimmten sensiblen Phasen gehäuft auftritt. Es entsteht ein Kreislauf, indem das Knibbelverhalten durch kurzfristige positive Konsequenzen bzw. den Wegfall negativer Zustände aufrechterhalten wird (s. D Abb. 1).

\section{Von der Angewohnheit zur Störung}

Langfristig gesehen folgen auf das Knibbeln jedoch negative Gefühle wie Ärger über die eigene Schwäche, Scham-, Schuldgefühle, niedriger Selbstwert sowie Unzufriedenheit mit dem eigenen Erscheinungsbild. Dementsprechend entsteht ein Teufelskreislauf aus

- kurzfristiger Entlastung,

- mittelfristig negativen Konsequenzen und

- erneuten Versuchen, diese negativen Gefühle durch das Knibbeln zumindest vorübergehend zu reduzieren.

Die langfristig negativen Konsequenzen überwiegen mit zunehmender Erkrankungsdauer deutlich, was zu signifikantem Leidensdruck und Beeinträchtigungen in wichtigen Funktionsbereichen führt.

Über klassische Konditionierungsprozesse können ursprünglich neutrale Kontextreize mit dem pathologischen Knibbeln assoziiert werden. Als konditionierte Reize lösen sie dann automatisch den Drang zum Knibbeln aus. Über Generalisierungsprozesse kann der Drang so in immer mehr Alltagssituationen auftreten. 


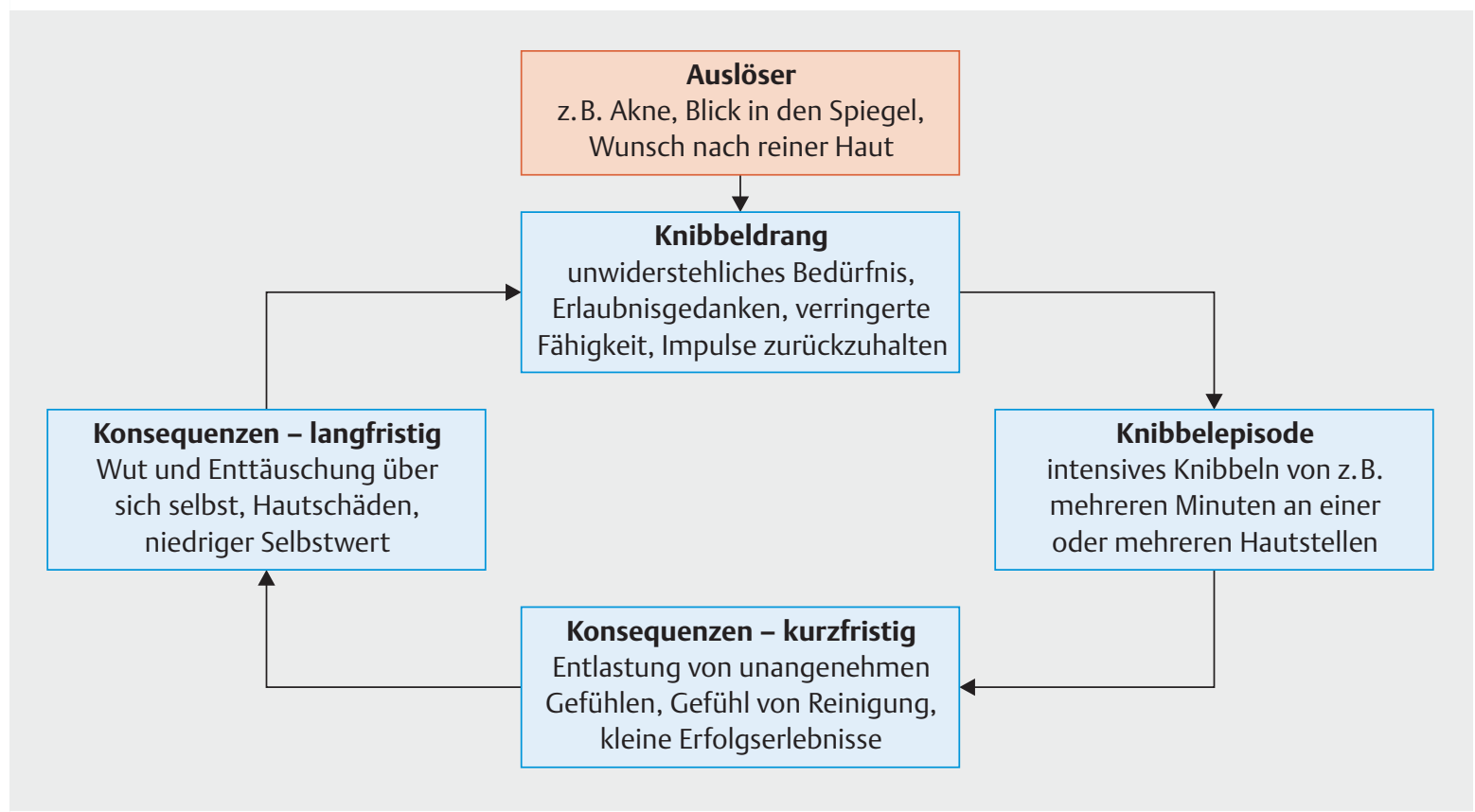

- Abb. 1 Teufelskreislauf bei Dermatillomanie.

\section{FALLBEISPIEL}

\section{Verhaltensanalyse nach Kanfer}

Situation

abends, allein im Bad, müde/erschöpft, Sorge über Konflikt am Arbeitsplatz, Ertasten von Hautunebenheiten beim Waschen, Anblick ungeschminkter Haut Organismus

junge Frau, perfektionistische Ansprüche, Bewertungsängste, leistungsabhängiger Selbstwert Reaktion

mit Fingern an Hautunregelmäßigkeiten herumdrücken, auf weitere Hautstellen ausdehnen, insgesamt ca. 15-20 Minuten; „Der Pickel sieht wirklich schlimm aus, den würden andere auch ausdrücken“,
„Jetzt sollte ich aufhören, aber diesen einen noch“; neutralisierte Gefühle; wie in Trance, ohne Zeitge-

fühl

\section{Konsequenzen}

- kurzfristig: erfolgreiches Ausblenden negativer Gefühle/Sorgen, körperliche Entspannung

- mittelfristig: Scham/Schuldgefühle, „Hoffentlich sieht das morgen keiner“, „An meinem Aussehen bin ich selbst schuld“, Ärger/Ekel über Hautschäden, Selbstabwertung

- langfristig: erneutes Aufkratzen von Wunden, Narbenbildung, geringes Selbstwertgefühl, sozialer Rückzug und vieles mehr
Hiermit ist auch unbewusstes, nicht zielgerichtetes Knibbeln erklärbar.

Dysfunktionale Kognitionen sind ebenfalls ein wichtiger Bestandteil bei der Erklärung der Dermatillomanie. Erlaubniserteilende Kognitionen wie „Nur ein Pickel, dann höre ich auf. " können den entscheidenden Startschuss für das problematische Verhalten geben. Auch dysfunktionale Überzeugungen wie „Ohne Knibbeln komme ich nicht zur Ruhe." halten die Angewohnheit möglicherweise aufrecht. Zusätzlich verursachen dysfunktionale Bewertungen mittelfristig Symptomstress, beispielsweise „Ich bin ekelhaft, weil ich so etwas mache.“ oder „Wenn die anderen mich so sehen, nimmt mich niemand mehr ernst.". Mit diesem typischen Auslöser für erneutes Knibbeln schließt sich ein Teufels- kreis, der mit jedem Umlauf an zerstörerischer Kraft gewinnt und den Ausstieg zunehmend schwierig macht.

\section{Behandlungsansätze}

Für Dermatillomanie liegen bisher nur wenige Behandlungsstudien vor [10]. Auch basieren die bisherigen Erkenntnisse auf Personengruppen mit verschieden definierten Kriterien für Dermatillomanie.

\section{Pharmakologische Behandlungsmethoden}

In einer Metaanalyse zeigte die Behandlung von Skin Picking mit SSRI und Lamotrigin keinen eindeutigen Vorteil gegenüber einer Placebobehandlung. Experimental- $(n=114)$, Placebo- $(n=47)$ und Wartekontroll- 
PRAXIS

In Studien untersuchte Medikamente

Folgende Medikamente wurden bisher für die Behandlung von Skin-Picking-Symptomen geprüft $[10,23]$.

\section{Open-Label- und randomisiert kontrollierte} Studien (RCT)

- Antidepressiva: selektive Serotonin-Wiederaufnahmehemmer (SSRI) und trizyklische Antidepressiva

- Antiepileptika: Lamotrigin

Fallstudien (Aussagekraft stark eingeschränkt)

- Opiatantagonisten: Naltrexon

- atypische Neuroleptika: Olanzapin

gruppe $(n=26)$ zeigten jedoch alle eine signifikante Verbesserung der Symptomatik über den zeitlichen Verlauf der Studien [10].

\section{Merke}

Die pharmakologische Behandlung von Skin-PickingSymptomen hat bisher keine eindeutige Überlegenheit gegenüber Placebomedikation gezeigt.

\section{Psychotherapeutische Behandlungsmethoden}

Verhaltenstherapeutische Interventionen bei Skin Picking zeigten sich einer inaktiven Kontrollgruppe überlegen [10]. Nach einer verhaltenstherapeutischen Intervention ( $n=151$ ) hat sich die Skin-Picking-Symptomatik signifikant verbessert. Behandlungen durch klinische Therapeuten und Selbsthilfeangebote zeigten keinen signifikanten Unterschied in ihrer Wirksamkeit [10].

\section{Merke}

Nach bisherigem Kenntnisstand sind bei Dermatillomanie nur kognitiv-verhaltenstherapeutische Interventionen überlegen gegenüber einer Nichtbehandlung.

\section{PRAXIS}

Verhaltenstherapeutische Behandlungsansätze mit nachgewiesener Wirksamkeit [10]

- Selbstbeobachtungstraining, Psychoedukation

- Strategien zur Reduktion des Knibbelns, z.B. Habit-Reversal-Training

- Rückfallprophylaxe

\section{Verhaltenstherapeutische Behandlungsbausteine}

Die folgenden verhaltenstherapeutischen Behandlungsbausteine basieren auf unseren eigenen Behandlungserfahrungen, einer Literaturrecherche zu Fallstudien [24] sowie der Sichtung von Behandlungsmanualen $[25,26]$ und Selbsthilfebüchern [24-28].

\section{Psychoedukation}

Die Aufklärung über Dermatillomanie als anerkanntes Störungsbild und epidemiologisches Wissen hilft, die Betroffenen zu entlasten und zu stärken. Mit ihnen wird ein individuelles Störungsmodell erarbeitet und der Teufelskreis des Knibbelns schlüssig nachvollzogen (s. Abb. 1). Daraus können unterschiedliche Behandlungsstrategien zur Durchbrechung des Kreislaufs abgeleitet und verständlich gemacht werden. Diese Wissensvermittlung kann die Veränderungsmotivation der Betroffenen, den Behandlungsverlauf und die Rückfallprophylaxe positiv beeinflussen.

\section{PRAXIS}

Ziele der Psychoedukation

- Entlastung durch Aufklärung über das Krankheitsbild

- Ableitung eines individuellen Störungsmodells mit entsprechenden Interventionsmöglichkeiten

\section{Selbstbeobachtungstraining}

Durch Selbstbeobachtung sollen die Betroffenen zu Experten ihres persönlichen Knibbelverhaltens werden. Sogenannte Knibbelanalysen helfen, Betroffenen vorausgehende und nachfolgende Kontextbedingungen, Gedanken und Gefühle ihres Knibbelns (Trigger und Konsequenzen) deutlich zu machen. Identifizierte Gesetzmäßigkeiten und Routinen werden später genutzt, um konkrete Strategien gegen das Knibbeln zu entwickeln. Die strukturierte Selbstbeobachtung kann in Form von Tagebüchern oder formalisierten „Knibbelprotokollen“ im Alltag dokumentiert werden. Solche Knibbelprotokolle stehen auf Anfrage bei der Erstautorin zur Verfügung.

\section{PRAXIS}

Selbstbeobachtungstraining

- Schulung der eigenen Wahrnehmung

- Beobachtung der Häufigkeit des Knibbelns, Trigger und Konsequenzen

- Ableitung von geeigneten Interventionen 


\section{Motivationsklärung und -aufbau}

Die Veränderungsmotivation der Betroffenen zu klären, fördern und stabilisieren, ist aufgrund des starken kurzfristigen Anreizcharakters des Knibbelns und der dadurch bedingten hohen Rückfallgefahr für einen (dauerhaften) Therapieerfolg entscheidend. Ziel ist es, eine bewusste Entscheidung für eine Verhaltensänderung zu treffen sowie individuelle Ziele zu formulieren. Das ehrliche Abwägen aller Vor- und Nachteile hilft den Betroffenen, eine realistische Entscheidung für (oder gegen) die Therapie zu treffen. Die Formulierung persönlicher Ziele dient dazu, Verbindlichkeit zu schaffen und den Therapieerfolg später einzuschätzen.

PRAXIS

Motivationsarbeit

- Kosten-Nutzen-Abwägung

- Aufbau einer stabilen Veränderungsabsicht

- Verbindlichkeit schaffen durch eigene Ziele

\section{Strategien zur Reduktion von Knibbeldrang und Knibbelverhalten}

Nach den ersten wichtigen Schritten werden nun darauf aufbauend konkrete Strategien zur Kontrolle des Knibbelns entwickelt und auf ihre Wirksamkeit getestet. Eine Strategie sorgfältig zu erproben, erfordert

- Zeit (mindestens 1 Woche mit konsequenter Anwendung),

- viele Wiederholungen (Übung) und

- Kreativität.

Cave

Häufigster Fehler ist das vorzeitige Verwerfen einer Strategie.

Verschiedene Varianten derselben Strategie sollten entwickelt und ausprobiert werden, um die individuelle Problematik exakt anzusprechen.

PRAXIS

Erprobte Behandlungsstrategien

- Stimuluskontrolle

- Habit-Reversal-Training (HRT; dt.: Training inkompatibler Reaktionen)

- kognitive Umstrukturierung

- Aufbau positiver Aktivtäten

\section{Stimuluskontrolle}

Ziel der Stimuluskontrolle ist es, typische Auslöser für das Knibbeln vorübergehend zu reduzieren, zu beseitigen oder zu vermeiden. Gleichzeitig sollten Aktivitä-

\section{PRAXIS}

\section{Tipps zur Stimuluskontrolle}

Erfolgreiche Stimuluskontrolle zeichnet sich aus

durch:

- Reduktion von typischen Auslösereizen und -situationen, z. B. Licht im Badezimmer dimmen oder Spiegel abhängen, um visuelle Trigger zu begrenzen

- Verhinderung des Knibbelns, z. B. Baumwollhandschuhe tragen

- Erhöhung der Aufmerksamkeit für unbewusstes Knibbeln, z. B. beim Fernsehschauen alle $5 \mathrm{Mi}-$ nuten einen Alarm stellen, der aktiv ausgeschaltet werden muss

Zusätzlich sollte die ausgewählte Strategie:

- realistisch und im Alltag leicht umsetzbar sein

- in sozialen Interaktionen möglichst nicht stören

ten und Dinge, die vor dem Knibbeln schützen, gezielt gestärkt werden. Die typischen Auslöser und Schutzfaktoren werden anhand des persönlichen Knibbelprotokolls (Selbstbeobachtungstraining) identifiziert.

Maßnahmen zur Stimuluskontrolle müssen individuell und an die veränderten Bedürfnisse der Betroffenen im Therapieprozess angepasst werden. Bereits bestehende Listen mit möglichst vielen Vorschlägen sowie ein gemeinsames Brainstorming helfen den Betroffenen dabei, die für sie jeweils erfolgversprechendsten Strategien auszuwählen (Beispiellisten auf Anfrage bei der Erstautorin).

\section{Merke}

Stimuluskontrolle ist eine temporäre Stütze, um eine erste Kontrolle über das scheinbar unkontrollierbare Knibbeln zu ermöglichen. Diese Maßnahmen sollte nach einer stabilen Phase der Abstinenz schrittweise wieder abgebaut werden!

\section{Habit-Reversal-Training (HRT)}

Das Habit-Reversal-Training (übersetzt: Gewohnheitsumkehr), kurz HRT, gibt es bereits seit 1973. Azrin und Nunn entwickelten HRT zur Behandlung erlernter, aber ungünstiger Verhaltensgewohnheiten. Mit HRT soll verhindert werden, dass eine unerwünschte Bewegung ausgeübt wird, indem man die antagonistische Muskulatur anspannt. Die wiederholte und regelmäBige Ausübung dieser inkompatiblen Reaktion soll zudem Anspannung abbauen und eine alternative Reaktion auf den Bewegungsimpuls etablieren: Die Verknüpfung zwischen Knibbelimpuls und Knibbelbewegung wird geschwächt. 


\section{Merke}

Eine effektive inkompatible Reaktion zum Knibbeln ist, die Hände mit auseinander gespreizten Fingern möglichst weit vom Körper weg zu strecken.

\section{Kognitive Umstrukturierung}

Bei Dermatillomanie haben Erlaubnisgedanken wie „Ohne Knibbeln geht's bei mir nicht.“ oder „Diesen Pickel würde jede ausdrücken.“ einen direkten, ungünstigen Einfluss auf das Verhalten. Gedanken wie "Jetzt sehe ich noch schlimmer aus als vorher." reduzieren den Selbstwert und können das Bedürfnis, zu knibbeln, indirekt fördern. Gedankenprotokolle machen die Betroffenen auf typische erlaubniserteilende und belastende Gedanken aufmerksam. Die dysfunktionale Wirkung dieser Gedanken wird reflektiert. Daraufhin gilt es, angemessenere und hilfreiche Gedanken zu entwickeln, welche die Selbstwirksamkeit und den Selbstwert stärken sowie die Stressbewältigung fördern.

\section{Merke}

Die kognitive Umstrukturierung unterstützt die Betroffenen darin, sich ihrer ungünstigen (automatischen) Gedanken bewusst zu werden und diese durch zielführende Bewertungen zu ersetzen.

\section{Aufbau positiver Aktivitäten}

Positive Aktivitäten können in schwierigen Situationen eine hilfreiche Alternative zum Knibbeln sein. Allgemein wirken sie gleichzeitig stimmungsverbessernd sowie stressausgleichend. Für einen langfristigen Therapieerfolg ist es wichtig, dem Knibbeln mit einer Vielzahl an alternativen befriedigenden Verhaltensweisen möglichst viel Konkurrenz zu machen. In Depressionsmanualen und -ratgebern gibt es eine Reihe von Listen mit einer Vielzahl positiver Aktivitäten zur Auswahl.

\section{PRAXIS}

\section{Einsatz positiver Aktivitäten}

Positive Aktivitäten helfen als Ersatz:

- bei akutem Knibbeldrang

- bei Anspannung und Stress

- bei Schlaflosigkeit am Abend

- um sich etwas Gutes zu tun

- als Belohnung

\section{Rückfallprophylaxe}

\footnotetext{
Merke

Eine gründliche Nachbereitung der erlernten Knibbelstoppstrategien sowie eine sorgfältige Vorbereitung auf zukünftige Schwierigkeiten sind für einen langfristigen Therapieerfolg unabdingbar.
}

\section{FALLBEISPIEL}

\section{Stimuluskontrolle bei Frau Z.}

Der Anblick des Gesichts im Spiegel ist für Frau Z. ein typischer Auslöser ihres übermäßigen Knibbelns. Im Rahmen der Stimuluskontrolle soll deshalb der Anblick der Haut im Bad verhindert werden. Frau Z. nimmt folgende Veränderungen vor:

- Linie auf dem Boden vor dem Waschbecken ziehen, die nicht überschritten werden darf, um die Haut nicht näher im Spiegel betrachten zu können

- Licht dimmen, Spiegel mit bunter Folie abkleben

- Kosmetikspiegel entfernen

- im Zimmer abschminken mit feuchten Tüchern

Zusätzlich hilft es Frau Z., wenn sie sich eine Stoppuhr stellt, um ihre Zeit im Badezimmer zu begrenzen und durch den Alarm ggf. aus einer Knibbel-„Trance“ aufgeschreckt zu werden. Um den Alarm im Flur auszustellen, muss sie ihr Knibbeln unterbrechen und das Badezimmer verlassen. Dies bietet ihr die Möglichkeit, die Knibbelhandlung bewusst wahrzunehmen und durch die Unterbrechung zu beenden.

\section{PRAXIS}

Ablauf des Habit-Reversal-Trainings (HRT)

- Auswahl einer inkompatiblen Reaktion (z. B. Finger spreizen oder zur Faust ballen)

" „Trockenübung“: inkompatible Reaktion in Situationen ohne Knibbelimpuls einüben, Muskelspannung mindestens 1 - 3 Minuten halten, mehrfache Wiederholung mit kurzen Lockerungsphasen

- Inkompatible Reaktion bei akutem Knibbeldrang konsequent einsetzen. Anwendung so lange wiederholen, bis der Drang zum Knibbeln abklingt.

- Sich selbst für die Anwendung der inkompatiblen Reaktion belohnen.

- Bei ausreichender Sicherheit in der Anwendung ggf. eine unauffälligere inkompatible Reaktion für die Öffentlichkeit einüben (z. B. Hände in den Hosentaschen zu Fäusten ballen).

Die Betroffenen sollten über das bestehende Rückfallrisiko aufgeklärt sein und im Umgang mit einem Rückfall trainiert werden. Dazu sind individuelle Risikosituationen für einen Rückfall in das alte Verhalten zu identifizieren und ein individueller Notfallplan zu erstellen.

\section{Prognose}

Eine deutliche Reduktion des Knibbelns oder sogar eine völlige Abstinenz sind realistische Therapieziele. Wichtigste Voraussetzung hierfür ist die individuelle Motivation und die Förderung der Bewältigungskompetenz der Betroffenen. Um das geänderte Verhalten langfristig aufrechtzuerhalten, bedarf es einer kontinuierli- 


\section{PRAXIS}

Inhalt für den individuellen Notfallplan

- Vorsorgestrategien, die auf frühe Anzeichen eines drohenden Rückfalls reagieren

- Bewältigungsstrategien für wiederkehrenden Knibbeldrang

- Bewältigungsstrategien für einen tatsächlichen Rückfall

chen Weiterarbeit und Rückfallvorsorge. Selbsthilfegruppen können dabei eine gute Unterstützungsfunktion einnehmen.

\section{Fazit und Perspektiven}

Dermatillomanie ist eine Krankheit, die für Betroffene mit hohem Leidensdruck und Einschränkungen einhergeht. Erste Forschungsarbeiten weisen auf eine wirksame Behandlung durch Verhaltenstherapie hin. Generell sind jedoch viele Bereiche der Dermatillomanie bisher nur ansatzweise untersucht.

\section{KERNAUSSAGEN}

- Dermatillomaniebetroffene leiden unter dem wiederkehrenden Drang, ihre Haut zu bearbeiten (drücken, quetschen, knibbeln). Der empfundene Drang, die erfolglosen Versuche, das Knibbeln zu unterlassen, sowie die daraus resultierenden Gewebeschäden führen zu Leiden und Beeinträchtigungen.

- Dermatillomanie gewinnt durch die Aufnahme in das DSM-5 an Bedeutung. Viele Aspekte dieses Störungsbilds sind noch nicht gründlich erforscht.

- Es gibt erste Ansätze eines Risikomodells, welches die Wahrscheinlichkeit für die Entstehung der Dermatillomanie erklären könnte.

- Betroffene wünschen sich spezifische und wirksame Behandlungsangebote.

- Kognitiv-verhaltenstherapeutische Behandlungsansätze sind bei Dermatillomanie wirksam.

- Für einen langfristigen Therapieerfolg bedarf es großer Motivation und sorgsamen Vorausplanens, um Rückfälle zu vermeiden.

\section{Interessenkonflikt}

Die Autoren geben an, dass kein Interessenkonflikt besteht.

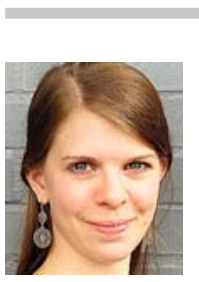

\section{Linda M. Mehrmann}

M. Sc. Psychologie. Jahrgang 1988. $2008-$ 2013 Bachelor- und Masterstudium Psychologie an der Universität Bielefeld. Seit 2013 Weiterbildung zur Psychologischen Psychotherapeutin (Schwerpunkt Verhaltenstherapie). Seit 2015 Wissenschaftliche Mitarbeiterin an der Universität zu Köln. Schwerpunkt der Promotionsarbeit: Dermatillomanie.

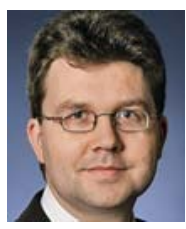

\section{Alexander L. Gerlach}

Prof. Dr. Jahrgang 1966. 1987 - 1994 Diplomstudium Psychologie, Universität Bamberg und Universität Marburg. 1994 - 1998 Promotionsstudium Psychologie, Universität Marburg und Stanford University. Habilitation 2005 an der Westfälischen Wilhelms-Universität Münster. Seit 2003 Psychologischer Psychotherapeut (Schwerpunkt Verhaltenstherapie). Seit 2004 staatlich anerkannter Supervisor. Seit 2009 Universitätsprofessor für Klinische Psychologie und Psychotherapie an der Universität zu Köln.

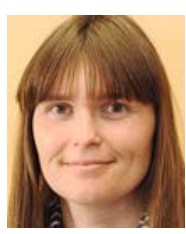

\section{Antje Hunger (geb. Bohne)}

Prof. Dr. Jahrgang 1972. 1992 - 1998 Diplomstudium Psychologie an der Universität Marburg. 1998- 2003 Promotionsstudium Psychologie, Universität Marburg und Massachusetts General Hospital/Harvard Medical School, Boston. 2003-2011 Wissenschaftliche Mitarbeiterin an der Westfälischen Wilhelms-Universität Münster. Seit 2006 Psychologische Psychotherapeutin (Schwerpunkt Verhaltenstherapie). Seit 2011 staatlich anerkannte Supervisorin. 2012 Vertretungsprofessorin an der FH Düsseldorf. Seit 2013 Professorin an der Hochschule Düsseldorf.

\section{Korrespondenzadresse}

\author{
Linda Mehrmann, M.Sc. Psychologie \\ Klinische Psychologie und Psychotherapie \\ Universität zu Köln \\ Pohligstraße 1 \\ 50969 Köln \\ E-Mail: linda.mehrmann@uni-koeln.de
}

Wissenschaftlich verantwortlich gemäß Zertifizierungsbestimmungen

Wissenschaftlich verantwortlich gemäß Zertifizierungsbestimmungen für diesen Beitrag ist Prof. Dr. Erwin Schultz, Nürnberg.

\section{Erstveröffentlichung}

Dieser Beitrag wurde erstveröffentlicht in: PSYCH up2date 2017; 11: $339-353$. 
[1] American Psychiatric Association, Falkai P, Wittchen HU, Hrsg. Diagnostisches und Statistisches Manual Psychischer Störungen DSM-5. 5. Aufl. Göttingen: Hogrefe; 2013

[2] Dilling H, Mombour W, Schmid MH. World Health Organization. Internationale Klassifikation Psychischer Störungen: ICD-10 Kapitel V (F, Klinisch-Diagnostische Leitlinien; Version 2006). Bern: Huber; 1991

[3] Saß H, Wittchen H-U, Zaudig M. DSM IV. Diagnostisches und Statistisches Manual Psychischer Störungen. Göttingen: Hogrefe; 1996

[4] Bohne A, Wilhelm S, Keuthen NJ et al. Skin picking in German students - prevalence, phenomenology, and associated characteristics. Behav Mod 2002; 26: 320-339

[5] Tucker BT, Woods DW, Flessner CA et al. The Skin Picking Impact Project: Phenomenology, interference, and treatment utilization of pathological skin picking in a populationbased sample. J Anxiety Disorders 2011; 25: 88 - 95

[6] Keuthen NJ, Deckersbach T, Wilhelm S et al. Repetitive skinpicking in a student population and comparison with a sample of self-injurious skin-pickers. Psychosomatics 2000; 41: $210-215$

[7] Odlaug BL, Grant JE. Clinical characteristics and medical complications of pathologic skin picking. Gen Hospital Psychiatry 2008; 30: 61-66

[8] Flessner CA, Woods DW. Phenomenological characteristics, social problems, and the economic impact associated with chronic skin picking. Behav Mod 2006; 30: 944 - 963

[9] Keuthen N], Deckersbach T, Wilhelm S et al. The Skin Picking Impact Scale (SPIS): Scale development and psychometric analyses. Psychosomatics 2001; 42: 397-403

[10] Schumer MC, Bartley CA, Bloch MH. Systematic review of pharmacological and behavioral treatments for skin picking disorder. J Clin Psychopharmacol 2016; 36: 147-152

[11] Snorrason I, Belleau EL, Woods DW. How related are hair pulling disorder (trichotillomania) and skin picking disorder? A review of evidence for comorbidity, similarities and shared etiology. Clin Psychol Rev 2012; 32: 618-629

[12] Odlaug BL, Lust K, Schreiber LR et al. Skin picking disorder in university students: Health correlates and gender differences. Gen Hosp Psychiatry 2013; 35: 168-173

[13] Mehrmann LM, Hunger A, Gerlach AL. Pathologisches Hautzupfen/-Quetschen (Skin Picking): Erste Ergebnisse zur Psychometrie störungsspezifischer Messinstrumente. Z Klin Psychologie Psychother 2017; 46: 23-31

[14] Gallinat C, Keuthen NJ, Backenstrass M. Ein Selbstbeurteilungsinstrument zur Erfassung von Dermatillomanie: Reliabilität und Validität der deutschsprachigen Version der Skin Picking Scale-Revised. PPmP Psychother Psychosom Medizinische Psychologie 2016; 66: 249-255
[15] First MB. Handbuch der Differenzialdiagnosen - DSM. Göttingen: Hogrefe; 2017

[16] Roos A, Grant JE, Fouche JP et al. A comparison of brain volume and cortical thickness in excoriation (skin picking) disorder and trichotillomania (hair pulling disorder) in women. Behav Brain Res 2015; 279: 255 - 258

[17] Grant JE, Odlaug BL, Hampshire A et al. White matter abnormalities in skin picking disorder: A diffusion tensor imaging study. Neuropsychopharmacol 2013; 38: 763 - 769

[18] Grant JE, Odlaug BL, Chamberlain SR. A cognitive comparison of pathological skin picking and trichotillomania. J Psychiatric Res 2011; 45: 1634-1638

[19] Keuthen N], Bohne A, Himle M, Woods DW. Advances in the Conceptualization and Treatment of Body-focused repetitive Behaviors. In: Ling BE, Hrsg. Obsessive compulsive Disorder Research. Hauppauge, NY: Nova Biomedical Books; 2005: 1 - 29

[20] Feusner JD, Hembacher E, Phillips KA. The mouse who couldn't stop washing: Pathologic grooming in animals and humans. CNS Spectrums 2009; 14: 503-513

[21] Jones BC, Little AC, Burt DM et al. When facial attractiveness is only skin deep. Perception 2004; 33: 569-576

[22] Magin P, Adams J, Heading G et al. 'Perfect Skin', the media and patients with skin disease: A qualitative study of patients with acne, psoriasis, and atopic eczema. Austr J Primary Health 2011; 17: $181-185$

[23] Gelinas BL, Gagnon MM. Pharmacological and psychological treatments of pathological skin-picking: A preliminary metaanalysis. J Obsessive Compuls Relat Disord 2013; 2: 167 175

[24] Capriotti MR, Ely LJ, Snorrason I et al. Acceptance-enhanced behavior therapy for excoriation (skin-picking) disorder in adults: A clinical case series. Cogn Behav Practice 2015; 22: $230-239$

[25] Bohne A. Trichotillomanie. Göttingen: Hogrefe; 2009

[26] Franklin ME, Tolin DF. Treating Trichotillomania: Cognitivebehavioral Therapy for Hairpulling and related Problems. New York: Springer Science \& Business Media; 2007

[27] Vollmeyer K, Fricke S. Die eigene Haut retten. Hilfe bei SkinPicking. Köln: Balance Ratgeber; 2012

[28] Claiborn J, Pedrick C. The Habit Change Workbook: How to break bad Habits and form good Ones. Oakland: New Harbinger Publications; 2001

Bibliografie

DOI https://doi.org/10.1055/s-0043-120953

Akt Dermatol 2017; 43: 477-491

(c) Georg Thieme Verlag KG Stuttgart · New York ISSN 0340-2541 


\section{Punkte sammeln auf CME.thieme.de}

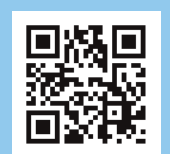

Diese Fortbildungseinheit ist 12 Monate online für die Teilnahme verfügbar.

Sollten Sie Fragen zur Online-Teilnahme haben, finden Sie unter http://cme.thieme.de|hilfe eine ausführliche Anleitung. Wir wünschen viel Erfolg beim Beantworten

der Fragen!

Unter https://eref.thieme.de/u2d003 oder über den QR-Code kommen Sie direkt zum Artikel zur Eingabe der Antworten.

VNR 2760512017152373561

\section{Frage 1}

Welche Aussage ist für Dermatillomanie zutreffend?

A Dermatillomanie beschreibt die normale menschliche Angewohnheit, die eigene Haut zu bearbeiten.

B Die Betroffenen empfinden keinerlei Leidensdruck oder Beeinträchtigung aufgrund des Knibbelns.

C Wenn Betroffene an Hautstellen knibbeln, wo es nicht öffentlich sichtbar ist, dann leiden sie auch nicht darunter.

D Betroffene sind hin und hergerissen zwischen dem befriedigenden Gefühl des Knibbelns und der Vermeidung der negativen Konsequenzen.

E Für Dermatillomanie wurden erstmals im DSM-IV explizite Kriterien definiert.

\section{Frage 2}

Wie wird das Störungsbild Dermatillomanie im DSM-5 charakterisiert?

A typische Zwangsgedanken bezogen auf das Zupfen/ Quetschen der Haut

B zwanghaftes Wiederholen bestimmter Bewegungsabläufe im Sinne einer Zwangshandlung

C übermäßige Beschäftigung mit einem oder mehreren wahrgenommenen Mängeln oder Defekten im äußeren Erscheinungsbild

D depressive Stimmung und Verlust von Freude und Interesse an anderen Aktivitäten außer Zupfen/Quetschen

E wiederkehrendes Zupfen/Quetschen der Haut, was Hautverletzungen zur Folge hat und trotz wiederholter Versuche nicht eingeschränkt oder unterlassen werden kann

\section{Frage 3}

Welche der folgenden Aussagen trifft nicht für Dermatillomanie zu?

A Es fällt Betroffenen schwer, über ihr Knibbelverhalten zu sprechen.

B Nur selten haben Betroffene Zugang zu spezifischen Behandlungsangeboten.

C Besonders häufig wird im Gesicht geknibbelt, es können aber auch andere Körperregionen betroffen sein.
D Betroffene schädigen ihre Haut, um Aufmerksamkeit von anderen zu erhalten.

E Das Knibbelverhalten kann auch unbewusst ausgelöst werden.

\section{Frage 4}

Welche der folgenden Aussagen stimmt nicht bezüglich der Epidemiologie von Dermatillomanie?

A Die Prävalenz für Dermatillomanie liegt schätzungsweise bei $1-5 \%$.

B Betroffene verheimlichen ihre Symptomatik häufig aufgrund von Schuld- und Schamgefühlen.

C Bisher wurden überwiegend weibliche DermatillomanieBetroffene erfasst.

D Der Erkrankungsbeginn liegt häufig zwischen dem 12. und 16. Lebensjahr.

E Das pathologische Knibbeln wird im Allgemeinen durch schwere Kindheitstraumata ausgelöst.

\section{Frage 5}

Was gehört nicht zum Teufelskreislauf der Dermatillomanie?

A Die Betroffenen verspüren ein unwiderstehliches Bedürfnis, die eigene Haut zu bearbeiten.

B Es kommt zu einer Episode intensiven Knibbelns an einer oder mehreren Hautstellen.

C Kurzfristige Konsequenzen sind eine Entlastung vom unangenehmen Dranggefühl verbunden mit einem kleinen Erfolgserlebnis.

D Langfristige Konsequenzen sind Wut und Enttäuschung über die vermeintlich eigene Schwäche sowie niedriger Selbstwert.

E Die wichtigste Konsequenz des Knibbelns ist, dass dadurch negative Gefühle dauerhaft bewältigt werden können. 


\section{Frage 6}

Behandlungsansätze: Welche Aussage ist richtig?

A Es liegen noch keine metaanalytischen Untersuchungen zur Wirksamkeit der Behandlung bei Dermatillomanie vor.

B Die Überlegenheit einer medikamentösen Behandlung der Dermatillomanie gegenüber einer Placebo-Behandlung ist nachgewiesen.

C Psychotherapie zeigte in Studien einen positiven Effekt auf die Knibbel-Symptomatik.

D Tiefenpsychologisch fundierte Interventionen wurden auf ihre Wirksamkeit bei Dermatillomanie empirisch überprüft.

E Eine therapeutische Intervention sollte bei Dermatillomanie immer auch medikamentös begleitet werden.

\section{Frage 7}

Was ist keine Funktion des Selbstbeobachtungstrainings?

A Die Offenlegung der Selbstbeobachtungsprotokolle gegenüber anderen Personen dient dem Abbau von Scham über dieses Verhalten.

B Die Selbstbeobachtung schult die eigene Wahrnehmung und schärft die Aufmerksamkeit für das unbewusste Knibbeln.

C Mithilfe der Selbstbeobachtung können die Häufigkeit des Knibbelns erfasst und Schwankungen gemessen werden.

D Die Selbstbeobachtung dient dem Erkennen von typischen Auslösern und Konsequenzen des Knibbelns.

E Aus den Selbstbeobachtungsprotokollen können geeignete Interventionsstrategien, beispielsweise für bestimmte Knibbelauslöser, abgeleitet werden.

\section{Frage 8}

Was ist eine geeignete inkompatible Reaktion zum Knibbeln?

A die Hände flach auf die Wangen legen

B an den Fingernägeln statt an Hautunreinheiten knibbeln

C die Hände vom Körper wegstrecken und die Finger spreizen

D laut in die Hände klatschen

E mit den Fingerspitzen die Kopfhaut abtasten und an den Haaren entlangfahren

\section{Frage 9}

Positive Aktivitäten helfen als Ersatz zum Knibbeln in unterschiedlichen Situationen. In welcher Situation sollten sie jedoch nicht eingesetzt werden?

A bei akutem Knibbeldrang

B als Belohnung nach einer Knibbelepisode

$C$ bei Anspannung und Stress

D bei Schlaflosigkeit am Abend

E um sich etwas Gutes zu tun

\section{Frage 10}

Welche Aussage zur Psychotherapie bei Dermatillomanie ist zutreffend?

A Motivationsklärung und -aufbau spielen in der Behandlung eine nebensächliche Rolle.

B Es ist wichtig, dass allgemeine, fest vorgegebene Stimuluskontrollstrategien von der betroffenen Person ausprobiert werden.

C Strategien zur Stimuluskontrolle sollten zügig verworfen werden, wenn sie nicht direkt eine positive Veränderung bewirken.

D In der Behandlung ist eine ehrliche Auseinandersetzung mit Erlaubnisgedanken und möglichen Alternativgedanken förderlich.

E Positive Aktivitäten können therapeutisch gut genutzt werden, wenn sie als Bestrafung für das Knibbeln entzogen werden. 